\title{
Construção de Domínios Bi-Scott via Completação por Ideais
}

R. CALLEJAS-BEDREGAL ${ }^{1}$, Departamento de Matemática, Universidade Federal da Paraíba, 58059-900 João Pessoa, PB, Brasil

B.R.C. BEDREGAL ${ }^{2}$, Laboratório de Lógica e Inteligência Computacional, Departamento de Informática e Matemática Aplicada, Universidade Federal do Rio Grande do Norte, 59072-970 Natal, RN, Brasil.

Resumo. Uma técnica para lidar com domínios não contáveis através de domínios contáveis é usar a noção de completação. Neste artigo, propomos uma representação de domínios bi-Scott (domínios de Scott cuja reversa também é um domínio de Scott) via completação por ideais de posets contáveis bi-finitamente consistentemente completos com menor e maior elemento. Mostramos que o construtor intervalar é fechado sobre esta classe de domínios e que funções monotônicas entre estes posets podem ser transformadas (completadas) numa função contínua entre os domínios bi-Scotts representados pelos posets. A partir deste último resultado, podemos obter uma definição básica de computabilidade entre domínios bi-Scott baseada na noção clássica de computabilidade (por exemplo, usando máquinas de Turing) entre seus posets de elementos finitos.

\section{Introdução}

A matemática intervalar, introduzida por R. Moore [10, 12], visa prover um controle dos erros computacionais que resultam de computações numéricas envolvendo representações finitas de números reais. A teoria original de Moore não somente considera intervalos reais, mas também intervalos complexos, matrizes de intervalos reais e complexos, etc. $[3,12,8]$. Assim, podemos ter intervalos de diversos tipos de dados, pelo que podemos pensar em intervalos paramétricos, o qual implica em ver intervalos como um construtor de tipos. Com este objetivo, em [4], foi introduzido um construtor intervalar sobre posets junto com uma categoria de poset, os domínios d-Scott (dcpos $\omega$-algébricos consistentemente completos cujos posets opostos também são dcpos $\omega$-algébricos consistentemente completos), fechada sobre este construtor. Neste artigo, consideraremos uma sub-categoria de d-Scott, chamada por nós de domínios bi-Scott, isto é domínios de Scott (cpos $\omega$-algébricos consistentemente completos) cujo poset oposto também é um domínio de Scott e que provamos ser fechada sobre o construtor intervalar.

\footnotetext{
${ }^{1}$ roberto@mat.ufpb.br

2 bedregal@dimap.ufrn.br
} 
Por outro lado, um ideal de um poset pode ser visto como a computação ou como o conjunto de aproximações de um objeto ideal. A completação por ideais de um poset adiciona estes objetos ideais ao poset. Em [13], foi provado que a completação por ideais de um poset resulta num cpo. Em [7], foi provado que a completação por ideais de uma pré-ordem resulta num cpo algébrico e que todo cpo algébrico é ordem-isomorfa à completação por ideais de seus elementos compactos. Em [17, 2] foi provado que a completação por ideais de um poset finitamente consistentemente completo, ou seja um poset em que todo conjunto finito majorado tem supremo, resulta em um domínio de Scott e que todo domínio de Scott é ordem isomorfa à completação por ideais de seus elementos compactos, que por sua vez são um poset finitamente consistentemente completo.

Neste artigo, introduziremos a noção de poset bi-finitamente consistentemente completo e mostraremos que a completação por ideais deste tipo de poset é um domínio bi-Scott. Também mostraremos que todo domínio bi-Scott é ordem isomorfa à completação por ideais de seu poset (bi-finitamente consistentemente completo) de elementos compactos e que funções bi-contínuas podem ser obtidas canonicamente a partir de funções monotônicas no poset de compactos, e vice-versa, ou seja, toda função monotônica nos compactos determina de maneira única uma função contínua. Este resultado é fundamental para se obter uma teoria da computabilidade sobre domínio bi-Scott (que considera universos contáveis e não contáveis) baseada simplesmente em computabilidade sobre o seus compactos, ou seja, sobre um universo contável, e portanto, onde pode ser considerada a noção de computabilidade de Church-Turing.

Neste artigo, estamos assumindo que o leitor tem conhecimento dos conceitos básicos de teoria dos domínios. Para uma leitura introdutória sobre este assunto, recomendamos os textos $[14,7,17]$.

\section{Representando Domínios de Scott via Comple- tação por Ideais}

Conjuntos munidos de uma ordem parcial, são chamados de posets. Seja $\langle P, \leq\rangle$ um poset e $X \subseteq P$. Um majorante de $X$ é qualquer elemento $m \in P$ tal que $x \leq m$ para cada $x \in X$. O menor de todos os majorantes de um conjunto $X$, se existir, é chamado de supremo de $X$ e é denotado por $\bigsqcup X . \Delta \subseteq P$ é um conjunto dirigido, se para cada $x, y \in \Delta$, existe um $z \in \Delta$ tal que $x \leq z$ e $y \leq z$. Dois posets, $\mathbf{P}_{1}=\left\langle P_{1}, \leq_{1}\right\rangle$ e $\mathbf{P}_{2}=\left\langle P_{2}, \leq_{2}\right\rangle$, são ordens isomorfas, denotado por $\mathbf{P}_{1} \cong \mathbf{P}_{2}$, se existe uma função bijetiva $\varphi: P_{1} \longrightarrow P_{2}$ tal que: $x \leq_{1} y$ se, e somente se, $\varphi(x) \leq_{2} \varphi(y)$. Um elemento $x \in P$ é compacto ou finito, se quando $x \leq \bigsqcup \Delta$, para um conjunto dirigido $\Delta \subseteq P$, existe um $y \in \Delta$ tal que $x \leq y$. Denotaremos por $\mathbf{P}^{0}$ o conjunto de todos os elementos compactos do poset $\mathbf{P}$. Note que, se o poset tem um menor elemento $\perp$, então ele é finito.

Um poset $\langle P, \leq\rangle$ com menor elemento $\perp$ é chamado de poset finitamente consistentemente completos (PFCC) se cada subconjunto finito não vazio de $P$ que é majorado (ou seja, que é consistente) tem supremo em $P$. 
Proposição 2.1. Sejam os posets $\mathbf{P}_{1}=\left\langle P_{1}, \leq_{1}\right\rangle$ e $\mathbf{P}_{2}=\left\langle P_{2}, \leq_{2}\right\rangle$ tais que $\mathbf{P}_{1} \cong \mathbf{P}_{2}$. Se $\mathbf{P}_{1}$ é um PFCC, então $\mathbf{P}_{2}$ também é.

Demonstração. Imediato.

Definição 2.1. Seja $\mathbf{P}=\langle P, \leq\rangle$ um poset. Um subconjunto não vazio $I \subseteq P$ é $u m$ ideal de $\mathbf{P}$ se $I$ é um conjunto dirigido e para cada $y \in I$, se $x \leq y$ então $x \in I$. Seja $\widehat{P}$ o conjunto dos ideais de $\mathbf{P}$. O poset $\widehat{\mathbf{P}}=\langle\widehat{P}, \subseteq\rangle$ é chamado de completação por ideais de $\mathbf{P}$.

Proposição 2.2. Seja $\mathbf{P}=\langle P, \leq, \perp\rangle$ um PFCC e $\Delta \subseteq \widehat{P}$.

1. Se $\Delta$ é dirigido em $\widehat{\mathbf{P}}$, então $\bigcup \Delta$ é um ideal de $\mathbf{P}$ e $\bigcup \Delta=\bigsqcup \Delta$.

2. $\cap \Delta$ é um ideal de $\mathbf{P}$.

Demonstração. 1. Seja $\Delta \subseteq \widehat{P}$ um conjunto dirigido. Então

- Se $x, y \in \bigcup \Delta$, então existem $X, Y \in \Delta$ tais que $x \in X$ e $y \in Y$. Portanto, por $\Delta$ ser dirigido, existe $Z \in \Delta$ tal que $X \subseteq Z$ e $Y \subseteq Z$. Logo, $x, y \in Z$. Como $Z$ é um ideal, existe $z \in Z$, e portanto $z \in \bigcup \Delta$, tal que $x \leq z$ e $y \leq z$.

- Se $y \in \bigcup \Delta$, então $\exists Y \in \Delta$ tal que $y \in Y$. Assim, se $x \leq y$, então, por $Y$ ser ideal, $x \in Y$. Portanto, $x \in \bigcup \Delta$.

Portanto, $\bigcup \Delta$ é um ideal.

Como a ordem em $\widehat{\mathbf{P}}$ é a inclusão, $\bigcup \Delta$ é um majorante de $\Delta$. Se $X$ é outro majorante de $\Delta$, então $I \subseteq X$ para cada $I \in \Delta$. Logo, $\bigcup \Delta \subseteq X$. Conseqüentemente, $\bigcup \Delta=\bigsqcup \Delta$.

2. Seja $\Delta \subseteq \widehat{P}$.

- Uma vez que $\perp$ está em qualquer ideal, $\bigcap \Delta \neq \emptyset$.

- Se $y \in \bigcap \Delta$, então $\forall Y \in \Delta, y \in Y$. Logo, se $x \leq y$, então $x \in Y$, pois $Y$ é um ideal. Portanto, $x \in \bigcap \Delta$.

- Se $x, y \in \bigcap \Delta$, então $\forall Z \in \Delta, x \in Z$ e $y \in Z$. Assim, por $Z$ ser um ideal, existe $z \in Z$ tal que $x \leq z$ e $y \leq z$. Como $\mathbf{P}$ é finitamente consistentemente completo e $\{x, y\}$ é consistente, $\{x, y\}$ tem supremo, ou seja, existe $w=\bigsqcup\{x, y\}$. Como cada $Z \in \Delta$ é um ideal e $x, y \in Z$, temos que $w \in Z$. Portanto, $w \in \bigcap \Delta$.

Logo, $\cap \Delta \in \widehat{P}$.

Para cada $x \in P$, o conjunto $\downarrow x=\{y \in P / x \leq y\}$ é um ideal, chamado de ideal principal gerado por $x$ [17].

A seguinte proposição mostra que os elementos finitos do poset $\widehat{\mathbf{P}}$, são exatamente os ideais principais gerados por elementos de $\mathbf{P}$. 
Proposição 2.3. Seja $\mathbf{P}=\langle P, \leq\rangle$ um poset com um menor elemento $\perp$. Então $\widehat{\mathbf{P}}^{0}=\{\downarrow x / x \in P\}$.

Demonstração. $(\Rightarrow)$ Seja $I$ um elemento compacto de $\widehat{\mathbf{P}}$. Como $I$ é um ideal, então $\downarrow I=\{\downarrow a / a \in I\}$ é um conjunto dirigido. Claramente, $I \subseteq \bigsqcup \downarrow I$ e, por $I$ ser compacto, existe um ideal $\downarrow x \in \downarrow I$ tal que $I \subseteq \downarrow x$. Logo, por definição de $\downarrow I$, $x \in I$ e portanto $\downarrow x \sqsubseteq I$. Conseqüentemente, $I=\downarrow x$.

$(\Leftarrow)$ Seja $x \in P$. Se $\Delta \subseteq \widehat{P}$ é um conjunto dirigido tal que $\downarrow x \subseteq \bigcup \Delta$, então $x \in I$ para algum $I \in \Delta$ e portanto $\downarrow x \subseteq I$. Logo, $\forall x \in P, \downarrow x$ é um elemento compacto de $\widehat{\mathbf{P}}$.

Lema 2.1. [9] Seja $\mathbf{P}=\langle P, \leq\rangle$ um poset contável com $\perp$ sendo o menor elemento. $O$ poset $\widehat{\mathbf{P}}=\langle\widehat{P}, \subseteq\rangle$ é um cpo $\omega$-algébrico.

Teorema 2.1. [18] Seja $\mathbf{P}=\langle P, \leq, \perp\rangle$ um PFCC contável. O cpo w-algébrico $\widehat{\mathbf{P}}=\langle\widehat{P}, \subseteq, \downarrow \perp\rangle$ é um domínio de Scott.

Observe que, em algum sentido, os elementos de um poset podem ser pensados como informações dos elementos de um domínio de Scott. Precisamente, aquele domínio obtido via completação por ideais daquele poset. Deste ponto de vista, cada elemento de um domínio de Scott pode ser visto como um conjunto de informações sobre ele. Note que os elementos finitos do domínio de Scott $\widehat{\mathbf{P}}$ são os ideais principais gerados pelos elementos do poset. Portanto, como será mostrado a seguir, os elementos finitos de um domínio de Scott constituem um poset finitamente consistentemente completo.

Lema 2.2. Seja $\mathbf{D}=\langle D, \sqsubseteq, \perp\rangle$ um domínio de Scott. Então $\mathbf{D}^{0}=\left\langle D^{0}, \sqsubseteq, \perp\right\rangle$ é um PFCC contável.

Demonstração. Por definição de domínio de Scott, $D^{0}$ é contável e $\perp \in D^{0}$.

Seja $S \subseteq D^{0}$, finito. Se $x \in D^{0}$ é um majorante de $S$ então, como $\mathbf{D}$ é consistentemente completo, $S$ tem supremo em D. Mostraremos que $\bigsqcup S \in D^{0}$. Seja $\Delta \subseteq D$ um conjunto dirigido. Se $\bigsqcup S \sqsubseteq \bigsqcup \Delta$ então, por transitividade, para cada $s \in S, s \sqsubseteq \sqcup \Delta$. Como cada $s \in S$ é finito, existe um $x_{s} \in \Delta$ (par cada $s \in S$ ) tal que $x_{s} \sqsubseteq s$. Portanto, pela propriedade de dirigido, existe um $z \in \Delta$ majorando todo esses $x_{s}$. Consequentemente, $z$ é também um majorante de $S$. Logo, $\bigsqcup S \sqsubseteq z$ para algum $z \in \Delta$ e portanto $\bigsqcup S$ é um elemento finito.

A idéia de se fazer a completação por ideais é sair de conjuntos não contáveis para contáveis. Mostraremos que a completação por ideais do PFCC dos elementos finitos de um domínio de Scott é um domínio de Scott, os quais são ordem-isomorfas.

Teorema 2.2. Todo domínio de Scott é ordem-isomorfa à completação por ideais de algum PFCC contável.

Demonstração. Seja $\mathbf{D}=\langle D, \sqsubseteq, \perp\rangle$ um domínio de Scott. Pelo lema 2.2 e por todo domínio de Scott ter um conjunto contável de elementos finitos, $\left\langle D^{0}, \sqsubseteq, \perp\right\rangle$ é um 
PFCC contável. Provaremos que a completação por ideais de $\mathbf{D}^{0}$ é ordem-isomorfa com D. Primeiro, seja $\varphi: D \longrightarrow \widehat{D^{0}}$ a função definida por

$$
\varphi(x)=\downarrow x \cap D^{0} .
$$

Essa função está, claramente, bem definida e é bijetiva. De fato, sua inversa é definida por $\varphi^{-1}(X)=\bigsqcup X$.

Assim, só resta mostrar que $\varphi$ é uma função monotônica.

Se $x$ e $y$ pertencem a $D$ e $x \sqsubseteq y$, então $\downarrow x \subseteq \downarrow y$ e portanto $\downarrow x \cap D^{0} \subseteq \downarrow y \cap D^{0}$, isto é, $\varphi(x) \subseteq \varphi(y)$. Logo, $\varphi$ é uma função monotônica.

Corolário 2.2.1. $\mathbf{D}=\langle D, \sqsubseteq, \perp\rangle$ é um dominio de Scott se, e somente se, $\mathbf{D}$ é ordem-isomorfa à completação por ideais de algum PFCC contável.

Demonstração. Segue dos teoremas 2.1 e 2.2 .

\section{Representando Domínios bi-Scott via Comple- tação por Ideais}

Um domínio bi-Scott é um domínio de Scott, isto é um cpo $\omega$-algébrico consistentemente completo, cujo poset oposto também é um domínio de Scott. Nesta seção, mostraremos que todo domínio bi-Scott pode ser obtido via completação por ideais de PFCC cujos oposto também são PFCC.

Definição 3.1. Seja $\mathbf{P}=\langle P, \leq\rangle$ um poset. O poset oposto ou reverso de $\mathbf{P}$ é o poset $\mathbf{P}^{o p}=\left\langle P^{o p}, \leq^{o p}\right\rangle$ onde $P^{o p}=P$ e $x \leq^{o p}$ y se, e somente se, $y \leq x$.

Definição 3.2. Seja $\mathbf{P}=\langle P, \leq\rangle$ um $P F C C$. $\mathbf{P}$ é chamado bi-finitamente consistentemente Completo (bi-PFCC) se $\mathbf{P}^{o p}$ também é um PFCC.

\section{Observações:}

1. A proposição 2.1 pode ser adaptada para bi-PFCC;

2. Cada poset bi-consistentemente completo (posets que tanto ele quanto seu oposto são consistentemente completos), também é bi-PFCC. Mas a reversa não é verdade. Um exemplo deste caso é o poset $\left\langle\mathbb{I}(\mathbb{Q})_{\perp}^{\top}, \sqsubseteq\right\rangle$ tal que $\mathbb{I}(\mathbb{Q})_{\perp}^{\top}=$ $\{[p, q] / p, q \in \mathbb{Q}$ e $p \leq q\} \cup\{\perp, \top\}$ e $X \sqsubseteq Y$ se, e somente se, $X=\perp, Y=\top$ ou $\left(\pi_{1}(X) \leq \pi_{1}(Y)\right.$ e $\left.\pi_{2}(Y) \leq \pi_{1}(X)\right)$, onde $\pi_{1}([p, q])=p$ e $\pi_{2}([p, q])=q$; e

3. Se $\mathbf{P}=\langle P, \leq\rangle$ é um poset com um maior e menor elemento, então $\mathbf{P}$ é biPFCC se, somente se, cada $X \subseteq P$ finito não vazio tem supremo e ínfimo, ou seja se $\mathbf{P}$ é um reticulado.

Lema 3.1. Seja $\mathbf{P}=\left\langle P, \leq, \perp, \perp_{o p}\right\rangle$ um bi-PFCC com $\perp$ e $\perp_{o p}$ como menores elementos de $\mathbf{P}$ e $\mathbf{P}^{o p}$, respectivamente. Ent $\tilde{a} o \widehat{\mathbf{P}}^{o p} \cong \widehat{\mathbf{P o p}}$. 
Demonstração. O isomorfismo é a função $f: \widehat{P^{o p}} \longrightarrow \widehat{P}^{o p}$ definida por

$$
f\left(I^{o p}\right)=\bigcap_{x \in I^{o p}} \downarrow x
$$

cuja inversa é a função $f^{-1}: \widehat{P}^{o p} \longrightarrow \widehat{P^{o p}}$ definida por

$$
f^{-1}(I)=\bigcap_{x \in I} \uparrow x .
$$

Mostraremos que estão bem definidas. Como $\perp \in \downarrow x$, para cada $x \in I^{o p}$, então $f\left(I^{o p}\right)$ é não vazio. Se $x, y \in f\left(I^{o p}\right)$, então para cada $z \in I^{o p}, x, y \in \downarrow z$. Logo, $x \leq z$ e $y \leq z$, portanto $f\left(I^{o p}\right)$ é um dirigido em $\mathbf{P}$. Se $x \in f\left(I^{o p}\right)$, então para cada $z \in I^{o p}, x \leq z$ e, portanto, se $y \leq x$, então também $y \leq z$. Logo, $y \in f\left(I^{o p}\right)$. Portanto, $f\left(I^{o p}\right) \in \widehat{P}=\widehat{P}^{o p}$. Analogamente, Como $\perp_{o p} \in \downarrow_{o p} x=\uparrow x$ para cada $x \in I$, então $f^{-1}(I)$ é não vazio. Se $x, y \in f^{-1}(I)$, então para cada $z \in I, x, y \in \uparrow z$. Logo, $z \leq x$ e $z \leq y$ ou, equivalentemente, $x \leq^{o p} z$ e $y \leq^{o p} z$. Portanto, $f^{-1}(I)$ é um dirigido em $\mathbf{P}^{o p}$. Se $x \in f^{-1}(I)$, então para cada $z \in I, x \leq^{o p} z$ e, portanto, se $y \leq^{o p} x$, então também $y \leq^{o p} z$. Logo, $y \in f^{-1}(I)$. Portanto, $f^{-1}(I) \in \widehat{P^{o p}}$.

A monotonicidade de $f$ e $f^{-1}$ é clara.

Teorema 3.1. Seja $\mathbf{P}=\left\langle P, \leq, \perp, \perp_{o p}\right\rangle$ um bi-PFCC com $\perp$ e $\perp_{\text {op }}$ sendo os menores elementos de $\mathbf{P}$ e $\mathbf{P}^{o p}$, respectivamente. Então $\widehat{\mathbf{P}}=\langle\widehat{P}, \subseteq, \downarrow \perp\rangle$ é um domínio biScott.

Demonstração. Pelo teorema 2.1, $\widehat{\mathbf{P}}$ é um domínio de Scott. Assim, só precisamos demonstrar que $\widehat{\mathbf{P}}^{o p}$ também é um domínio de Scott.

Como $\mathbf{P}^{o p}$ também é um PFCC com menor elemento, então, pelo teorema 2.1, sua completação por ideais, isto é $\widehat{\mathbf{P}^{o p}}$, é um domínio de Scott. Mas, pelo lema 3.1, $\widehat{\mathbf{P}}^{o p}$ é ordem isomorfa a $\widehat{\mathbf{P}^{o p}}$. Portanto, $\widehat{\mathbf{P}}^{o p}$ também é um domínio de Scott.

\section{Completação de Funções Monotônicas}

Definição 4.1. Sejam $\mathbf{P}_{1}$ e $\mathbf{P}_{2}$ posets e $f: P_{1} \longrightarrow P_{2}$ uma função monotônica. $A$ Completação de $f$ é a função $\widehat{f}: \widehat{P_{1}} \longrightarrow \widehat{P_{2}}$ definida por

$$
\widehat{f}(I)=\bigcup_{x \in I} \uparrow f(x) .
$$

Claramente, a função $\widehat{f}$ está bem definida e é monotônica.

Teorema 4.1. Sejam $\mathbf{P}_{1}$ e $\mathbf{P}_{2}$ PFCCs e $f: P_{1} \longrightarrow P_{2}$ uma função monotônica. Então, $\widehat{f}: \widehat{P_{1}} \longrightarrow \widehat{P_{2}}$ é uma função contínua.

Demonstração. Seja $\Delta \subseteq \widehat{P_{1}}$ um conjunto dirigido. Então, $\bigsqcup \Delta=\bigcup \Delta$. Logo,

$$
\begin{aligned}
\widehat{f}(\bigcup \Delta) & =\bigcup_{x \in \bigcup \Delta} \uparrow f(x) \\
& =\bigcup\{\uparrow f(x) / x \in I \text { e } I \in \Delta\} \\
& =\bigcup\left\{\bigcup_{x \in I} \uparrow f(x) / I \in \Delta\right\} \\
& =\bigcup\{\widehat{f}(I) / I \in \Delta\} \\
& =\bigcup \widehat{f}(\Delta) .
\end{aligned}
$$


Portanto, $\widehat{f}$ é contínua.

\section{Construtor Intervalar sobre Domínios bi-Scotts}

Seja $\mathbb{I}(\mathbb{R})=\{[r, s] / r, s \in \mathbb{R}$ e $r \leq s\}$ o conjunto dos intervalos reais. Cada intervalo pode ser visto como um conjunto $([r, s]=\{x \in \mathbb{R} / r \leq x \leq s\})$ ou como um par ordenado. A teoria de Moore [10, 11, 12] garante que a maioria das operações e funções usuais para os números reais podem ser estendidas de modo natural para $\mathbb{I}(\mathbb{R})$, a partir dos extremos dos intervalos [15]. Diversas ordens parciais naturais podem ser definidas sobre $\mathbb{I}(\mathbb{R})[4]$. Uma delas é a ordem

$$
[a, b] \sqsubseteq[c, d] \Leftrightarrow a \leq c \leq d \leq b,
$$

chamada de informação, introduzida por Dana Scott, em [16], e usada por Benedito Acióly, em [1], para proporcionar uma fundamentação computacional da matemática intervalar.

Note que tanto a definição de intervalo real, quanto a ordem de informação, dependem da ordem usual de $\mathbb{R}$. Callejas-Bedregal e Bedregal, em [4], generalizaram esta construção considerando qualquer conjunto parcialmente ordenado.

Definição 5.1. Seja $\mathbf{D}=\langle D, \leq\rangle$ um poset. O poset $\mathbb{I}(\mathbf{D})=\langle\mathbb{I}(D)$, $\rangle$, onde

- $\mathbb{I}(D)=\{[a, b] / a, b \in D$ e $a \leq b\}$

- $[a, b] \sqsubseteq[c, d] \Leftrightarrow a \leq c$ e $d \leq b$

é denominado poset dos intervalos de $\mathbf{D}$.

Em [4], foram mostradas diversas subcategorias de POSET que são fechadas para este construtor intervalar. Uma das características principais, destas subcategorias, é o fato de que se um posets era um objeto da categoria, então o seu oposto também era e de que não exigiam a existência de menor (e portanto de maior) elemento. Dentre todas as sub-categorias para as quais o construtor intervalar é fechado, está a categoria dos domínios d-Scott (dcpos $\omega$-algébricos consistentemente completos cujo poset oposto também é um dcpo $\omega$-algébrico consistentemente completo) com funções contínuas como morfismo. Note que todo domínio bi-Scott é um d-domínio, mas o contrário não é verdade, pois domínios d-Scott não precisam ter menores nem maiores elementos. Assim, trivialmente podemos construir, a partir de um domínio d-Scott, um domínio bi-Scott adicionando a ele um $\perp$ e um $T$. Observe também que domínios bi-Scott são reticulados $\omega$-algébricos cujo reticulado oposto também é algébrico [5]. O construtor intervalar quando aplicado a um domínio bi-Scott em geral não resulta num domínio bi-Scott. Por este motivo, neste artigo mudaremos sutilmente a definição do construtor intervalar.

Definição 5.2. Seja $\mathbf{D}=\langle D, \leq, \perp, \top\rangle$ um poset com menor e maior elemento. $O$ poset $\mathbb{I}(\mathbf{D})_{\perp}^{\top}=\left\langle\mathbb{I}(D)_{\perp}^{\top}, \sqsubseteq, \perp, \top\right\rangle$, onde

- $\mathbb{I}(D)=\{[a, b] / a, b \in D-\{\perp, \top\}$ e $a \leq b\}$ 
- $\mathbb{I}(D)_{\perp}^{\top}=\mathbb{I}(D) \cup\{\perp, \top\}$

- $\forall[a, b],[c, d] \in \mathbb{I}(D), \sqsubseteq[c, d] \Leftrightarrow a \leq c$ e $d \leq b$

- $\forall X \in \mathbb{I}(D)_{\perp}^{\top}, \perp \sqsubseteq X$ e $X \sqsubseteq \top$

é denominado poset dos intervalos de $\mathbf{D}$ estendido.

Proposição 5.1. Seja $\mathbf{D}=\langle D, \leq, \perp, \top\rangle$ um domínio bi-Scott. Então, $\mathbb{I}(\mathbf{D})_{\perp}^{\top}$ é um dominio bi-Scott.

Demonstração. É uma extensão natural do fechamento dos domínio d-Scott com respeito ao construtor intervalar [4], considerando que todo domínio d-Scott com menor e maior elemento é um domínio bi-Scott.

Outras propriedade que poderiam ser mostradas válidas são:

1. $\mathbb{I}\left(D^{0}\right)_{\perp}^{\top}=\left(\mathbb{I}(D)_{\perp}^{\top}\right)^{0}$, ou seja, os elementos finitos de $\mathbb{I}(\mathbf{D})_{\perp}^{\top}$ são intervalos de elementos finitos de $\mathbf{D}$.

2. $I$ é um ideal de $\mathbb{I}(\mathbf{D})_{\perp}^{\top}$ se, e somente se, $\pi_{1}(I)$ é um ideal de $\mathbf{D}^{o p}$ e $\pi_{2}(I)$ é um ideal de $\mathbf{D}$, onde $\pi_{1}(I)=\{\underline{x} /[\underline{x}, \bar{x}] \in I\}$ e $\pi_{2}(I)=\{\underline{x} /[\bar{x}, \bar{x}] \in I\}$.

3. Sejam os bi-PFCC's $\mathbf{P}_{1}$ e $\mathbf{P}_{2}$. Se $f: P_{1} \longrightarrow P_{2}$ é uma função monotônica, então $\mathbb{I}(f): \mathbb{I}\left(P_{1}\right)_{\perp}^{\top} \longrightarrow \mathbb{I}\left(P_{2}\right)_{\perp}^{\top}$, definida por

$$
\mathbb{I}(f)([\underline{x}, \bar{x}])=[f[\underline{x}), f(\bar{x})]
$$

é uma função monotônica. Portanto, a partir de uma função monotônica $f: D^{0} \longrightarrow E^{0}$, podemos obter uma função contínua entre $\mathbb{I}(\mathbf{D})_{\perp}^{\top}$ e $\mathbb{I}(\mathbf{E})_{\perp}^{\top}$.

\section{Computabilidade sobre Domínios bi-Scott}

Nesta seção, introduziremos uma noção de computabilidade para domínios bi-Scott baseada sobre a noção clássica de computabilidade (Church-Turing) aplicada ao poset de seus elementos finitos, que é um PFCC contável. A noção dada aqui é diferente da abordagem usual de domínios para este assunto (domínios efetivamente dados $[16,18,17])$. Isto, em princípio, proporcionaria uma nova abordagem para computabilidade no contínuo.

Definição 6.1. Sejam $\mathbf{D}_{1}$ e $\mathbf{D}_{2}$ domínios bi-Scotts e $f: D_{1} \longrightarrow D_{2}$ uma função contínua. $f$ é Scott-Computável (com respeito a $\mathbf{D}_{1}$ e $\mathbf{D}_{2}$ ), se existe uma função monotônica $g: D_{1}^{0} \longrightarrow D_{2}^{0}$ tal que

$$
f=\varphi_{D_{2}}^{-1} \circ \widehat{g} \circ \varphi_{D_{1}},
$$

onde $\varphi_{D_{i}}$ é a função definida na equação 2.1 para o domínio de Scott $\mathbf{D}_{i}$. 
Por exemplo, seja $f: \mathbb{N} \longrightarrow \mathbb{N}$ uma função Church-Turing computável. Então, a função $F: \wp(\mathbb{N}) \longrightarrow \wp(\mathbb{N})$, definida por $F(X)=\{f(x) / x \in X\}$, é Scottcomputável com respeito ao domínio bi-Scott $\left\langle{ }^{\wp}(\mathbb{N}), \subseteq, \emptyset, \mathbb{N}\right\rangle$. De fato,

$$
F=\varphi_{\wp_{(\mathbb{N})^{0}}^{-1}}^{-1} \widehat{F^{0}} \circ \varphi_{\wp}(\mathbb{N})^{0},
$$

onde $F^{0}: \wp(\mathbb{N})^{0} \longrightarrow \wp(\mathbb{N})^{0}$ é definido por $F^{0}\left(\left\{x_{1}, \ldots, x_{n}\right\}\right)=\left\{f\left(x_{1}\right), \ldots, f\left(x_{n}\right)\right\}$. Note que $\wp(\mathbb{N})^{0}=\{X \subseteq \mathbb{N} / X$ é finito $\}$.

Assim, esta noção de computabilidade estende a computabilidade tipo 1 (computabilidade clássica sobre conjuntos contáveis) para uma computabilidade tipo 2 (computabilidade sobre conjuntos não contáveis [19]).

\title{
7. Considerações Finais
}

Neste artigo propusemos uma construção dos aqui chamados domínios bi-Scott, através da completação por ideais do poset de seus elementos finitos. Este resultado é importante, uma vez que nos permite lidar com domínios eventualmente não contáveis e que incluem elementos "infinitos" (ideais), através de seus elementos finitos e que formam um poset contável. De fato, isto possibilitou introduzir uma noção de computabilidade sobre domínios bi-Scott baseada na noção usual de computabilidade sobre seus elementos finitos. Assim, este resultado nos permitiria afirmar, por exemplo, que a função $f: \wp(\mathbb{N}) \longrightarrow \wp(\mathbb{N})$, definida por $f(X)=\left\{x^{2} \in \mathbb{N} / x \in X\right\}$, é computável, pois ela, quando restrita aos conjuntos finitos, é computável. Outro resultado importante foi mostrar que esta classe de domínios é fechada sobre o construtor intervalar e, portanto, suporta semântica para o tipo de dados intervalos parametrizado.

\begin{abstract}
A technique to deal with non countable domains through of countable domains is to use a completion notion. In this paper we propose a representation for bi-Scott domain (Scott domains whose reverse also is a Scott domain) via ideal completion of bi-finitely consistent complete countable posets with lesser and greatest element. We show that the interval constructor is closed under this class of domains and that monotonic functions between this posets can be transformed (completed) in a continuous function between the bi-Scott domains represented by the posets. From this last result we can obtain a basic definition of computability between bi-Scott domains based on the classic computability notion (e.g. using Turing machines) between their respective poset of finite elements.
\end{abstract}

\section{Referências}

[1] B.M. Acióly, "Fundamentação Computacional da Matemática Intervalar", Tese de doutorado, CPGCC da UFRGS, Porto Alegre, 1991.

[2] B.R.C. Bedregal, "Sistemas de Informação Contínuos: Uma Abordagem Lógica e Computacional para a Matemática Intervalar", Tese de doutorado, DI-UFPE, Recife, 1996. 
[3] R. Boche, "Complex Interval Arithmetic whit Some Applications", Lockheed Missiles and Space Company, Technical Report LMSC4-22-66-1, Palo Alto, CA, 1965.

[4] R. Callejas-Bedregal, B.R.C. Bedregal, Interval as a Domain Constructor, em "Seleta do XXIII CNMAC" (E.X.L. de Andrade et al., eds.), Tendências em Matemática Aplicada e Computacional, Vol. 2, pp. 43-52, SBMAC, 2001.

[5] G. Gierz, K.H. Hofmann, K. Keimel, M. Mislove, D.S. Scott, "A Compendium of Continuous Lattices", Springer-Verlag, 1980.

[6] C.A. Gunter, Comparing categories of domains, in LNCS, Vol. 239, pp. 101121, Springer-Verlag, 1985.

[7] C.A. Gunter, "Semantics of Programming Languages: Structures and Techniques", MIT Press, 1992.

[8] L. Jaulin, M. Kieffer, O. Didrit, E. Walter, "Applied Interval Analysis", Springer-Verlag, London, 2001.

[9] J.D. Lawson, The versatile of continuous order, in LNCS, Vol. 298, SpringerVerlag, 1987.

[10] R.E. Moore, "Automatic error analysis in digital computation", Lockheed Missiles and Space Co. Technical Report LMSD-48421, Palo Alto, CA, 1959.

[11] R.E. Moore, "Interval Analysis", Pretince Hall, New Jersey, 1966.

[12] R.E. Moore, "Methods and Applications for Interval Analysis", Philadelphia, SIAM, 1979.

[13] G. Markowsky, B.K. Rosen, Bases for Chain-Complete Posets, IBM Journal of Research and Development, 20 (1976), 138-147.

[14] G. Plotkin, "Domains", Department of Computer Science, University of Edinburgh, 1983.

[15] R.H.N. Santiago, B.R.C. Bedregal, B.M. Acióly, Interval Representations, em "Seleta do XXVI CNMAC" (E.X.L. de Andrade et al., eds.), TEMA Tendências em Matemática Aplicada e Computacional, Vol. 5, No. 2, pp. 317326, SBMAC, 2004.

[16] D.S. Scott, Outline of a Mathematical Theory of Computation, in $4^{\text {th }}$ Annual Princeton Conference on Information Sciences and Systems, pp. 169-176, 1970.

[17] A. Stoltenberg-Hansen, I. Lindström, E.B. Griffor, "Mathematical Theory of Domains", Cambridge Tracts in Theoretical Computer Science 22, Cambridge university Press, 1994.

[18] M. Smyth, Effectively given domains. Theoretical Computer Science, 5 (1977), 257-274.

[19] K. Weihrauch, "Computable Analysis", Springer, Berlin, 2000. 